\title{
Pleomorphic Undifferentiated Sarcoma (Malignant Fibrous Histiocytoma) of True Vocal Fold: A Rare Laryngeal
} Malignancy

Rohit Bhardwaj, ${ }^{1}$ Ankur Gupta, ${ }^{1}$ Sabarirajan Ponnusamy, ${ }^{1}$ Karthika Nathan ${ }^{1}$

\begin{abstract}
$\underline{\text { Introduction }}$
Pleomorphic undifferentiated sarcoma also known as Malignant fibrous histiocytoma, is a malignanat tumour which commonly involves upper and lower extremities and the retroperitoneum. Only a few sporadic MFHs located in the head and neck have been reported in the literature. Rarely the tumor can involve larynx. Complete excision of the tumor is the standard treatment but post-operative chemotherapy and / or radiotherapy is also recommended in selected cases.

Case Report

We present a rare case report of pleomorphic undifferentiated sarcoma also known as malignanat fibrous histiocytoma of true vocal fold in a 65 years old male patient. The patient presented to us with complaints of change in voice for past 3 months. He also reported about his smoking habits for past 35 years. On detailed examination of head and neck region no palpable lymph nodes were detected. Oral and nasal cavity examination also did not reveal any pathology. On endoscopic laryngeal assessment a polypoidal mass was seen arising from posterior half of anterior third of left true vocal fold. The vocal fold mobility was found normal. We completely excised the tumor by microlaryngeal surgery. The histopathological and immunohistochemical examination of the excised tissue identified the tumor being a pleomorphic undifferentiated sarcoma which was positive for Vimentin, EMA and negative for CK, p63. Patient did not receive radiotherapy/ chemotherepy in postoperative period. Patient has been under follow-up for past 6 months and is disease free as of now.

Discussion

Mesenchymal malignancies of larynx are rare entities. Pleomorphic undifferentiated sarcoma also known as malignanat fibrous histiocytoma is one of such malignancy. The treatment consists of complete excision of the lesion. The need of postoperative radiotherapy and/ chemotherapy depends on the extent of disease.

$\underline{\text { Kevwords }}$

Histiocytoma, Malignant Fibrous; Sarcoma, Pleomorphic Undifferentiated; Vocal Cords
\end{abstract}

$\mathrm{M}$ esenchymal malignant tumors involving larynx are not so common. Pleomorphic undifferentiated sarcoma also known as Malignant fibrous histiocytoma (MFH) is one such mesenchymal malignancy, which commonly involves upper and lower extremities and the retroperitoneum. ${ }^{1}$

1 - Department of ENT, Vardhman Mahavir Medical College \& Safdarjung Hospital, New Delhi

\section{Corresponding author:}

Dr Ankur Gupta

email: drankurguptaent@gmail.com
Laryngeal involvement is rare and only few cases of vocal fold involvement have been reported in literature. ${ }^{2,3}$ Rarely these tumors are also reported to occur in patients who received radiation treatment for squamous cell carcinoma of the larynx. ${ }^{4}$ It originates in the interstitial cells that differentiate fibroblasts and histiocytes. ${ }^{5}$

The age group affected most commonly ranges between 50 and 70 years. ${ }^{6}$ The first-line treatment option for these sarcomas is surgical resection, with or without postoperative chemo- and/or radiotherapy. ${ }^{7}$ The disease has occasional metastasis and frequent recurrences. ${ }^{8} \mathrm{We}$ present a rare case report of pleomorphic undifferentiated sarcoma also known as malignant fibrous histiocytoma 


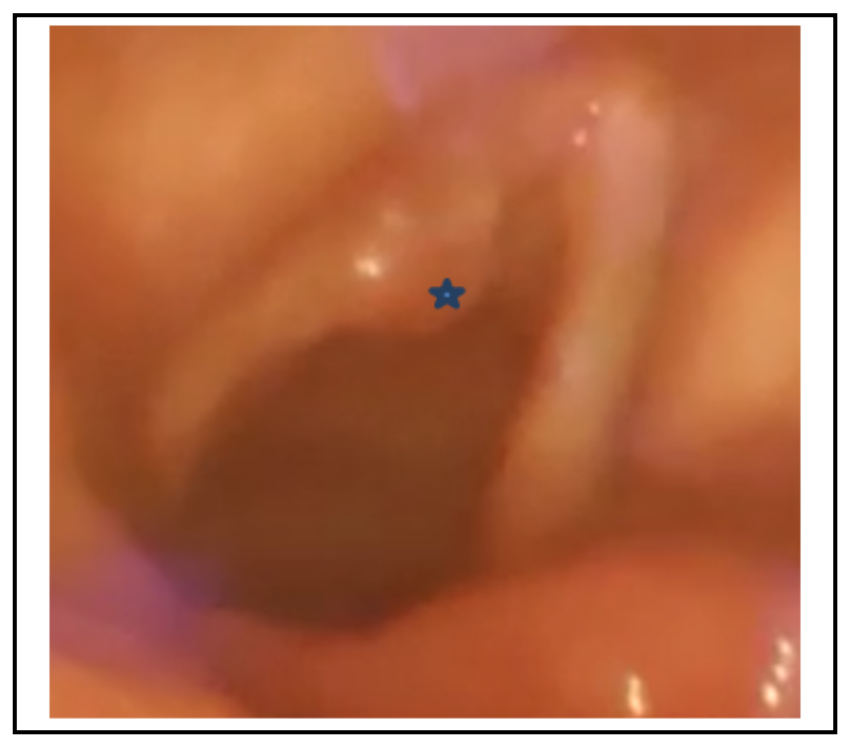

Fig.1. Preoperative endoscopic picture showing broad based polyp shaped vocal mass seen arising from posterior half of anterior third of left true vocal fold (star)

of true vocal fold in a 65 years old male patient.

\section{Case Report}

A 65 years old male patient, presented to ENT OPD with chief complaints of change in voice since past 3 months. Patient denied about complaints of difficulty in breathing, difficulty/ pain while swallowing, trauma to neck and recent neck surgery. Patient has been occasional smoker for past 35 years. On detailed examination of head and neck region, no palpable lymph nodes were detected. Oral and nasal cavity examination also did not reveal any pathology. On endoscopic laryngeal assessment, a polypoidal mass seen arising from posterior half of anterior third of left true vocal fold. The vocal fold mobility was found normal.

The diagnosis was considered as a large vocal cord polyp, considering the size, shape and site of origin. During micro laryngeal surgery for excision of the lesion, we felt the lesion as firm to hard in consistency and not soft as usually seen in vocal polyp. It was broad based and found firmly attached to the vocal fold (Fig.1). Thus, considering the suspicion of malignancy, the lesion was resected completely along with part of

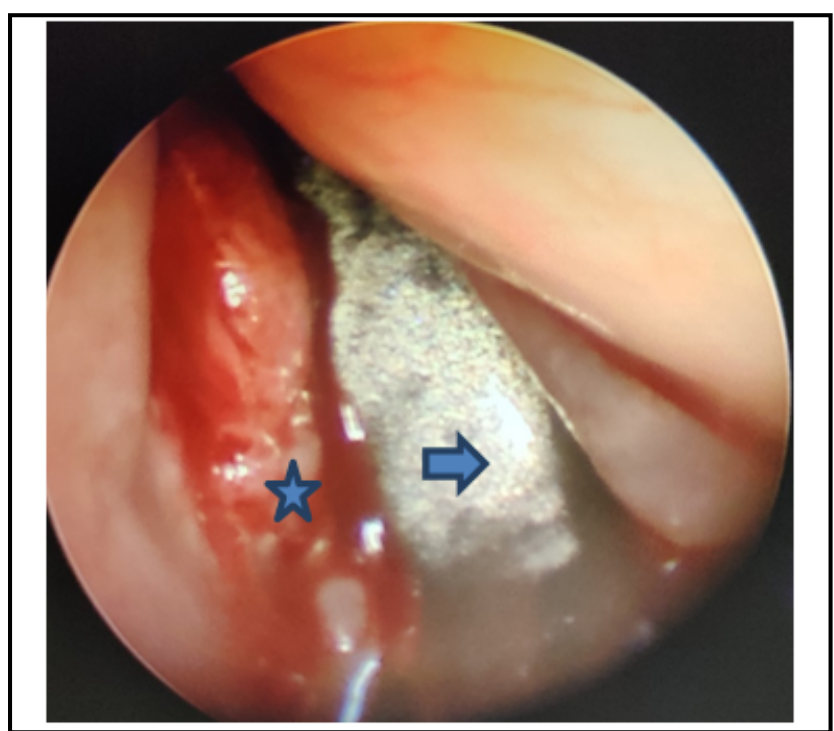

Fig.2. Intraoperative photograph showing the vocal fold after resection of the lesion from left true vocal fold ( star), endotracheal tube (arrow)

vocal fold under microscopic vision (Fig.2), keeping an adequate safety margin of approximately $2-3 \mathrm{~mm}$.

The postoperative histopathological and immunohistochemical examination of the excised tissue identified the tumor being a pleomorphic undifferentiated sarcoma (Fig.3) which was positive for Vimentin, EMA and CD68. It was negative for CK, p63, CD34, CD99, S-100 and Desmin. Also, the margin of the resected tissue found negative for disease.

The patient was further evaluated for metastatic spread of disease but found free of it. He did not receive radiotherapy/chemotherapy post operatively. The patient has been under our follow up for past 1 year and is free of residual / recurrent disease (Fig.4).

\section{Discussion}

As per WHO update on sarcoma, Malignant fibrous histiocytoma can be categorized as a pleomorphic undifferentiated sarcoma after ruling out other forms of differentiation. ${ }^{9}$ Malignant fibrous histiocytoma (MHF) is an older term but still widely used for these tumors. It is an aggressive tumor and treatment options include surgical resection, with or without postoperative chemo- 


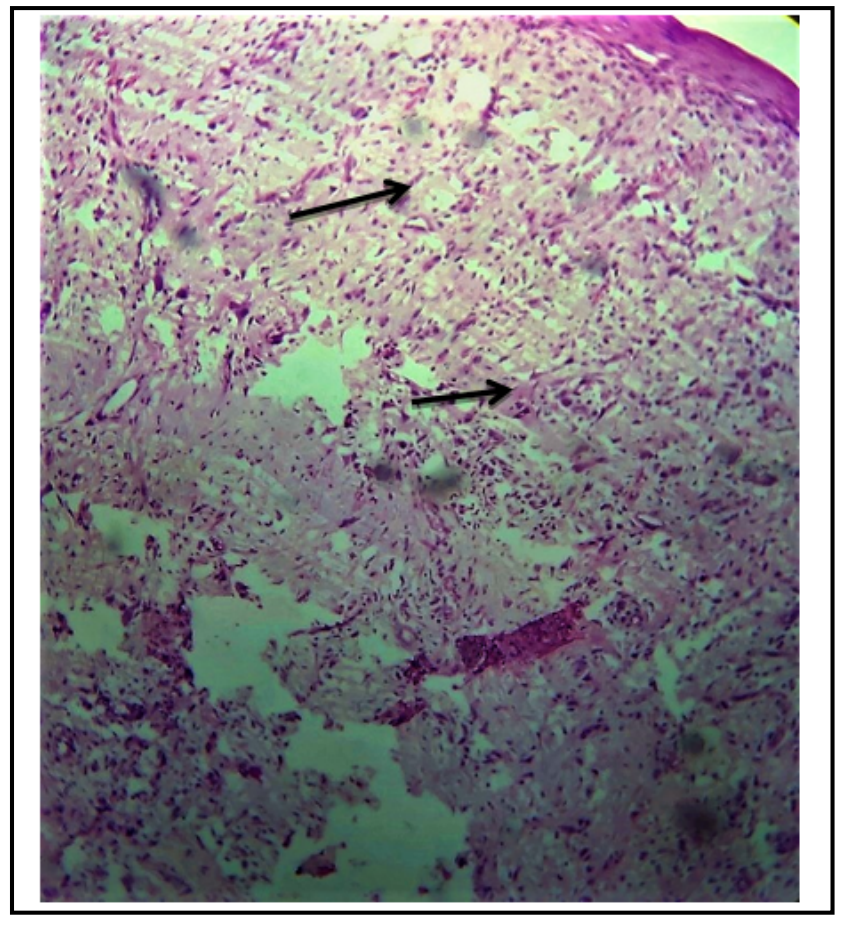

Fig. 3. Histopatologic picture of the lesion showing characteristic tangled (storiform) arrangement of the fibroblasts like spindle cells seen in malignant fibrous histiocytoma ( H\&E, 10X)

and/or radiotherapy. ${ }^{7}$ The recurrence rates are reported as high as $44 \%$, and rates of metastasis to the lungs and the lymph nodes are 82 and $32 \%$, respectively. ${ }^{10,11}$ The treatment plan and outcome depends to a great extent on the size, depth of involvement, and the inflammatory component of the tumor. ${ }^{10,11}$ On microscopic examination the tumor may appear as a vocal polyp or papilloma. Thus, diagnosis is usually made by histopathologic and immunohistochemical examination of the surgically resected tissue. Imaging studies also play a limited role in smaller tumors.

Complete surgical resection is the first line treatment. The surgeon should aim at achieving an adequate surgical margin without sacrificing important structures during excision, in order to avoid the risk of recurrence. Macroscopic/ microscopic observation during surgery should not be relied upon completely to decide extent of the resection. Ideally, histopathology should confirm margins to be safe. In some reported cases of Malignant fibrous histiocytoma of the larynx, the patients

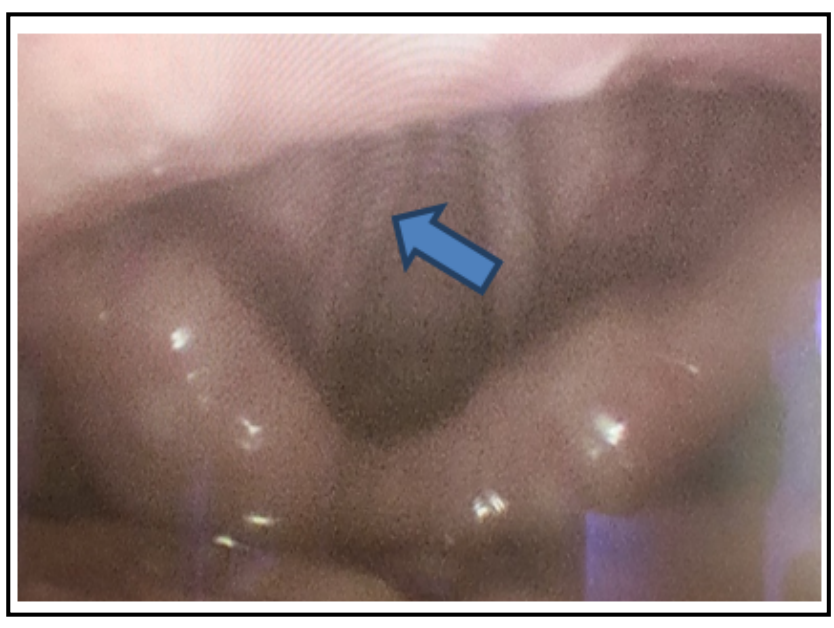

Fig. 4. Post-operative laryngeal endoscopy image well Healed normal left true vocal fold (arrow)

underwent total laryngectomy. ${ }^{12}$ In cases of incomplete resection, post-operative radiotherapy / chemotherapy should be considered. ${ }^{11,13}$ Local radiotherapy and adjuvant chemotherapy can significantly increase survival rates and reduce the risk of metastasis in these mesenchymal tumors. The treatment outcomes also have varied depending on the tumor's specific location. ${ }^{1}$

In our case where the tumor size was small (HPE specimen measured $0.7 \times 0.5 \times 0.5 \mathrm{~mm})$ and surgical margins were free of tumor, the risk of recurrence is minimized.

By this case study, we aim to stress the fact that a benign looking polyp like lesion which is firm in consistency, having a broad based attachment, in elderly age group, might indeed harbor a rare mesenchymal malignancy. Such lesions should be dealt more radically (complete surgical resection, taking adequate surgical margins) rather than a ligament sparing approach of true benign lesions, considering the higher rate of recurrence and much higher potential for distant metastasis. The need for post-operative radiotherapy and/or chemotherapy should be considered for selected cases after carefully examining the histopathology for completeness of resection.

\section{References}

1. Weiss SW, Enzinger FM: Malignant fibrous histiocytoma: An 
analysis of 200 cases. Cancer 1978; 41:2250-66

2. Masuda K, Takimoto T, Yashizaki T, et al. Malignant fibrous histiocytoma arising from the vocal cord. ORL J Otorhinolaryngol Relat Spec. 1989; 51(6):365-8

3. Khmel'nitskaia NM, Stepanova IuE, Iurkov IuA. Malignant histiocytoma of the larynx [in Russian]. Vestn Otorinolaringol. 2002; (5):46-8

4. Guney E, Yigitbasi OG, Balkanli S, Canoz OM: Postirradiation malignant fibrous histiocytoma of the larynx: a case report. Am J Otolaryngol. 2002; 23(5):293-6

5. O'Brien JE, Stout AP. Malignant fibrous xanthomas. Cancer 1964; 17:1445-55

6. Pathrose G, John NT, Manojkumar R. A rare case of malignant fibrous histiocytoma/pleomorphic undifferentiated sarcoma of the kidney. J Clin Diagn Res. 2015; 9(1):PD27-9

7. Patel SG, Shaha AR, Shah JP. Soft tissue sarcomas of the head and neck: An update. Am J Otolaryngol. 2001; 22(1):2-18
8. Sabesan T, Xuexi W, Yongfa Q, et al. Malignant fibrous histiocytoma: Outcome of tumours in the head and neck compared with those in the trunk and extremities. Br J Oral Maxillofac Surg. 2006; 44(3):209-12

9. Fletcher CD. The evolving classification of soft tissue tumours: An update based on the new WHO classification. Histopathology 2006; 48(1):3-12

10. Weiss SW, Enzinger FM. Myxoid variant of malignant fibrous histiocytoma. Cancer 1977; 39(4):1672-85

11. Zhang GB, Li J, Zhang PF, et al. Radiation-induced malignant fibrous histiocytoma of the occipital: A case report. World J Surg Oncol. 2014;12:98

12. Soh KB, Westmore GA, Moir AA, Colloby PS. Malignant fibrous histiocytomas of the larynx- Report of two cases. Ann Acad Med Singapore 1996; 25(6):878-81

13. Lewis JJ, Brennan MF. Soft tissue sarcomas. Curr Probl Surg. 1996; 33(10):817-72. 